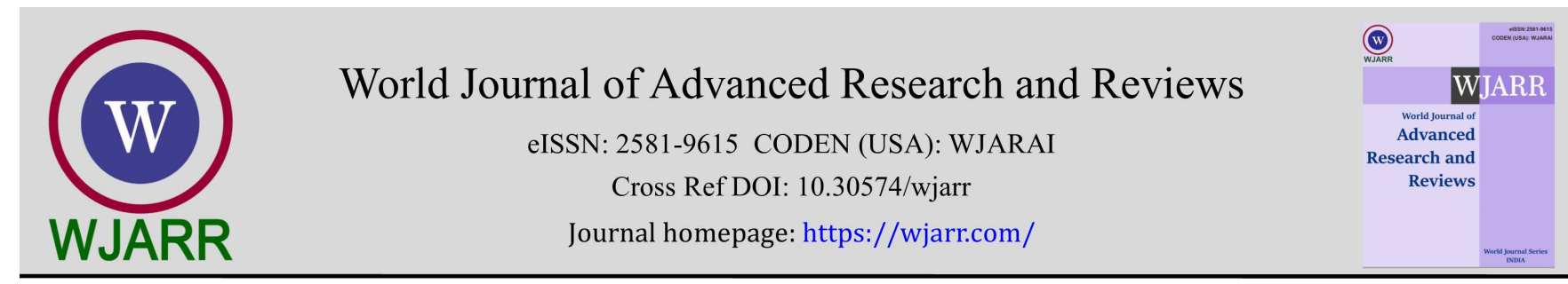

(REVIEW ARTICLE)

\title{
Prevention and treatment of COVID - 19: A review
}

\author{
T Peter ${ }^{1,{ }^{*}}$ and Ajithadas Aruna ${ }^{2}$ \\ ${ }^{1}$ Institute of Pharmacology, Madurai Medical College, Madurai - 20, India. \\ ${ }_{2}^{2}$ JDME, Directorate of Medical Education, Chennai -10, India.
}

World Journal of Advanced Research and Reviews, 2021, 09(03), 272-280

Publication history: Received on 05 February 2021; revised on 06 March 2021; accepted on 09 March 2021

Article DOI: https://doi.org/10.30574/wjarr.2021.9.3.0090

\begin{abstract}
Coronavirus, are belonging to the family Coronaviridae. Corona viruses have enveloped virions (virus particles) that measure approximately $120 \mathrm{~nm}(1 \mathrm{~nm}=10-9$ meter $)$ in diameter. Coronaviridae is generally considered to contain two genera, Coronavirus and Torovirus. It was the first coronavirus to be isolated from domestic fowls in the late 'known as SARS corona virus. COVID-19 is a disease caused by a new strain of corona virus. 'CO' stands for corona, 'VI' for virus, and 'D' for disease. Formerly, this disease was referred to as '2019 novel corona virus' or '2019-nCoV. Its origins have been traced to a food market in Wuhan, China, in December 2019.A virus apparently closely related to SARS corona virus. Symptoms begin 2 to 14 days after you come into contact with the virus. Early studies show that many people who have mild infections recover within 2 weeks. More severe cases tend to last 3 to 6 weeks. India is the fourth worsthit nation by the pandemic after the US, Brazil and Russia. There's currently no treatment specifically approved for COVID-19, and no cure for an infection, although treatments and vaccines are currently under study. Instead, treatment focuses on managing symptoms as the virus runs its course.
\end{abstract}

Keywords: 2019-nCoV; SARS corona virus; Prevention and treatment; Kaba Sura Kudineer

\section{Introduction}

Coronavirus, are belonging to the family Coronaviridae. Coronaviruses have enveloped virions (virus particles) that measure approximately $120 \mathrm{~nm}(1 \mathrm{~nm}=10-9$ meter $)$ in diameter. Club-shaped glycoprotein spikes in the envelope give the viruses a crown like, or coronal, appearance. The nucleocapsid, made up of aprotein shell known as a capsid and containing the viral nucleic acids, is helical or tubular. The coronavirus genome consists of a single strand of positivesense RNA (ribonucleic acid) “(1)”.

Coronaviridae is generally considered to contain two genera, Coronavirus and Torovirus, however, it can also be divided into alpha, beta, gamma, and delta.

Coronavirus (CoV) are infecting humans and variety of avian and mammalian species in the world, such as cats, birds, camels, dogs, bats, mice, and livestock"(2)".

In humans, a species known as SARS corona virus (or severe acute respiratory syndrome corona virus) causes a highly contagious respiratory disease that is characterized by symptoms of fever, cough, and muscle ache, often with progressive difficulty in breathing.

\footnotetext{
*Corresponding author: T Peter

Institute of Pharmacology, Madurai Medical College, Madurai - 20, India.
}

Copyright (c) 2021 Author(s) retain the copyright of this article. This article is published under the terms of the Creative Commons Attribution Liscense 4.0. 
In 2012 another coronavirus capable of causing a severe acute respiratory illness later known as Middle East respiratory syndrome (MERS) was discovered in humans.

The first case was found in Saudi Arabia, and others were reported within the following year in France, Germany, Jordan, Qatar, Tunisia, the United Arab Emirates, and the United Kingdom. All confirmed cases were directly or indirectly linked to the Middle East of all confirmed cases documented by 2019, roughly one-third had ended in death"(4)".

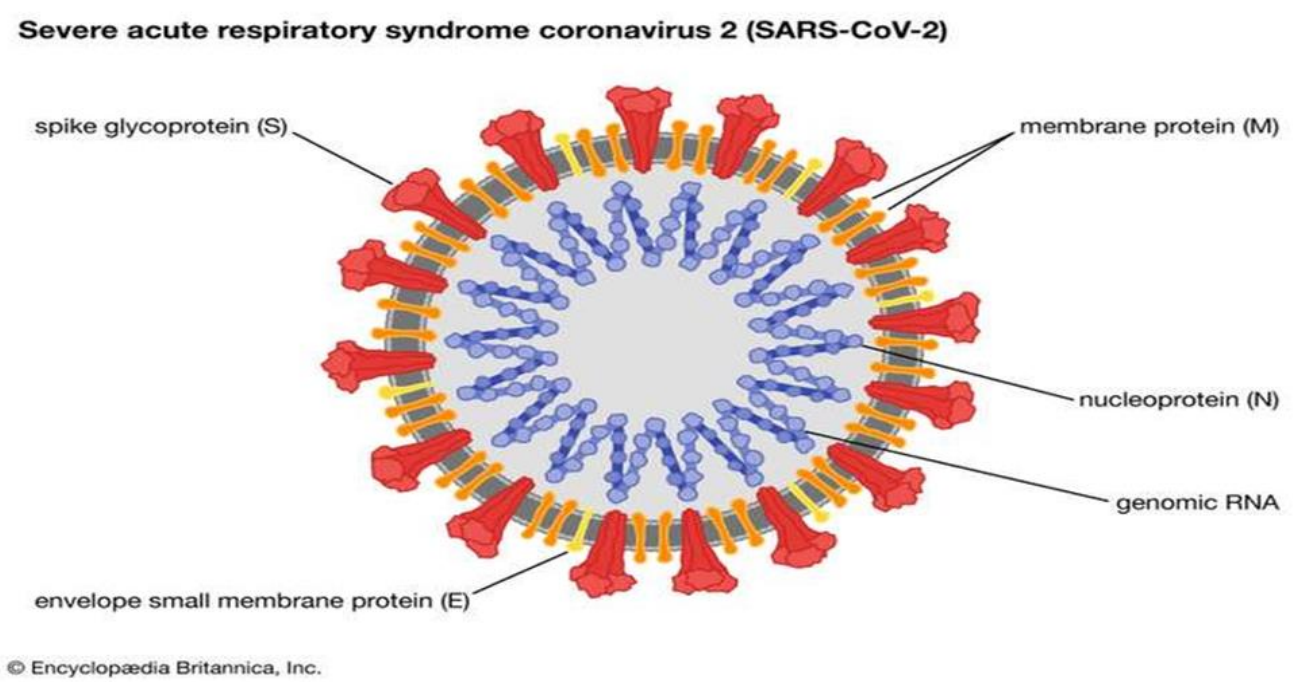

Figure 1 SARS-CoV-2

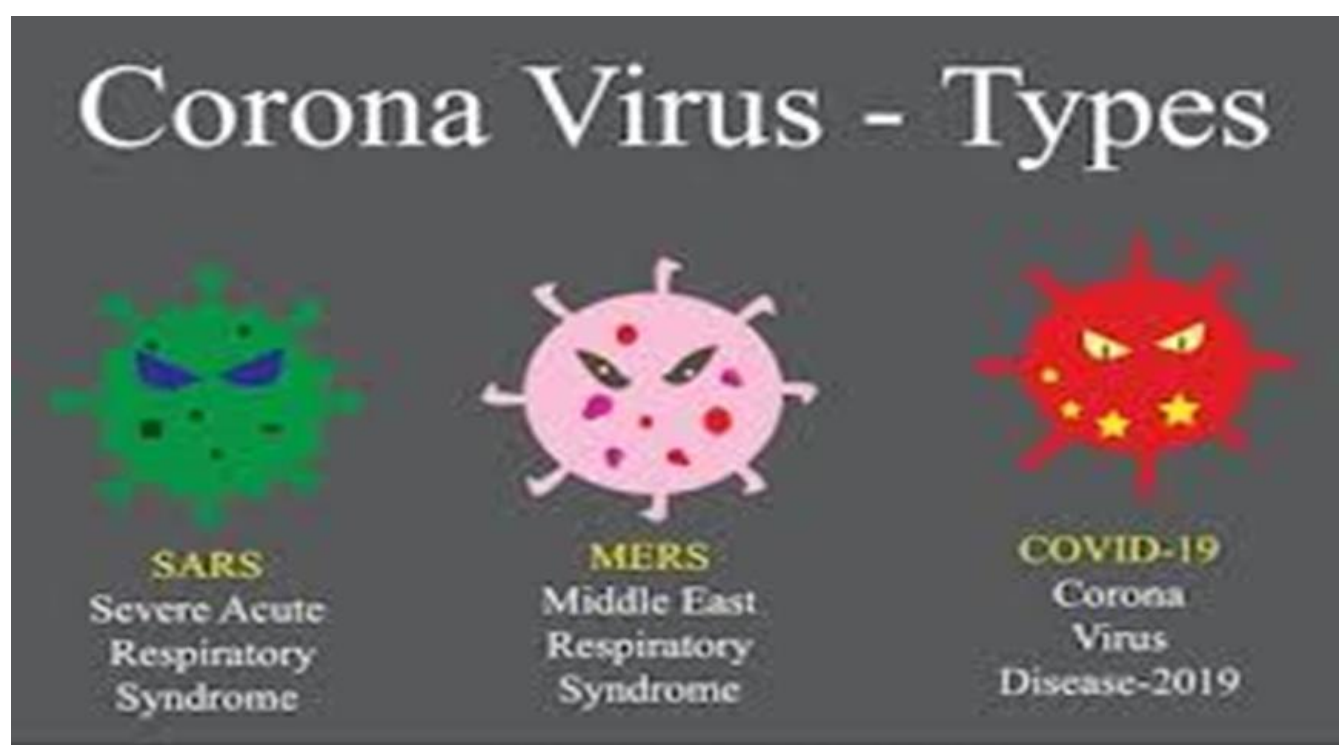

Figure 2 Types of coronavirus

The novel MERS coronavirus was similar to other coronaviruses known to have originated in bats and was thought to be passed from bats to other animals before being transmitted to humans. Camels were identified as one possible reservoir for the MERS virus"(1)".

\subsection{Origin and spread}

COVID-19 is a disease caused by a new strain of corona virus. 'CO' stands for corona, 'VI' for virus, and 'D' for disease. Formerly, this disease was referred to as '2019 novel corona virus' or '2019-nCoV'. 
Its origins have been traced to a food market in Wuhan, China, in December 2019.A virus apparently closely related to SARS corona virus. From there 2020, it's reached countries as distant as the United States and the Philippines and Europe, having been carried by travelers from affected regions.

In March the World Health Organization declared the outbreak a pandemic, and travel to, from, and within many countries was severely restricted in an effort to control its spread. In many areas, schools and many businesses closed, and stay-at-home guidelines were implemented, which strongly encouraged people not to leave their places of residence"(5)".

\subsection{Epidemiology}

As of March 10, 2020, the WHO has reported that there are 113,702 confirmed cases globally, and 4012 deaths have been registered; $71 \%$ of all confirmed cases $(80,924)$ and $78 \%$ of all deaths related to COVID-19 (3140) are from China and its territories. Since the first reported case in Wuhan, 109 other countries have declared that they have at least one confirmed case of COVID-19. The WHO has officially classified China as a "very high risk" region for COVID-19.

India is the fourth worst-hit nation by the pandemic after the US, Brazil and Russia. According to the Johns Hopkins University, which has been compiling COVID-19 data from all over the world, India is at the eighth position in terms of the death toll of the total 12,573 deaths"(6)".

\subsection{Clinical symptoms of COVID-19}

Symptoms begin 2 to 14 days after you come into contact with the virus. Early studies show that many people who have mild infections recover within 2 weeks. More severe cases tend to last 3 to 6 weeks.

Talk to your doctor about how long you should isolate yourself if you have symptoms. CDC guidelines say you can leave isolation when all of these are true:

- You haven't had a fever for 3 days.

- Your respiratory symptoms, such as coughing or shortness of breath, are better.

- Sneezing

- Bronchiolitis, which causes swelling in the small air sacs in the lungs

- Pneumonia, a lung infection

- Pain or pressure in your chest

- Confusion or severe drowsiness

- A blue tint to your lips or face

- Appetite loss

- A weak immune system

- Tell them if you are older than 60 or have another health condition, like diabetes, heart disease, or cancer.

- It's been at least 10 days since your symptoms began OR you have two negative COVID-19 tests 24 hours apart" $(1,4)^{\prime \prime}$.

\subsection{Patients tested for SARS-CoV-2}

Samples from the upper respiratory tract are used to test for the virus, using a blood, saliva, or tissue sample. However, most tests use a cotton swab to retrieve a sample from the inside of your nostrils. Polymerase chain reaction (PCR) is used to identify their viral RNA. If the test is positive, the diagnosis of SARS-CoV-2 is confirmed. Negative tests with a strong suspicion, such as clinical symptoms or exposure, can be repeated using samples from other respiratory sites"(7)".

\section{Sources and selection criteria}

A literature review was performed using Pub Med and Google to identify relevant English language articles published 2020. Search terms included coronavirus, severe acute respiratory syndrome coronavirus 2, 2019-nCoV, SARS- CoV-2, SARS- CoV, MERS CoV, and COVID-19 in combination with treatment and pharmacology. Additional relevant articles were identified from the review of citations referenced. 


\subsection{Review of Selected Repurposed Drugs}

Agents previously used to treat SARS and MERS are potential candidates to treat COVID-19. Various agents with apparent in vitro activity against SARS-CoV and MERS-CoV were used during the SARS and MERS outbreaks, with inconsistent efficacy.

\section{Prevention of infection}

The best way to prevent the transmission of infection is to avoid or limit contact with people who are showing symptoms of COVID-19 or any respiratory infection.

The next best thing you can do is practice good hygiene and physical distancing to prevent bacteria and viruses from being transmitted.

The CDC recommends multiple steps to prevent the transmission and risk of SARS-CoV-2. Frequent hand washing lasting at least 20 seconds by using soap and water is advised.

Hand sanitizers with at least $60 \%$ alcohol can also be used as an alternative.

The public has also been told to avoid touching mucosal surfaces such as the mouth and the nose with hands that have not been washed. Anyone showing symptoms of the virus should try to seek appropriate medical help.

They should also limit their exposure to other unaffected people and cover their noses and mouths when coughing or sneezing.

They are also advised to wear a facemask if they present with symptoms. You can make your own mask using basic materials such as:

- Bandana

- T-Shirt

- cotton fabric

Cloth masks are preferred for the general public since other types of masks should be reserved for healthcare workers.

It's critical to keep the mask clean. Wash it after each time you use it. Avoid touching the front of it with your hands. Also, try to avoid touching your mouth, nose, and eyes when you remove it.

This prevents you from possibly transferring the virus from a mask to your hands and from your hands to your face.

Keep in mind that wearing a mask isn't a replacement for other preventive measures, such as frequent hand washing and practicing physical distancing, all of them are important.

Certain people shouldn't wear face masks, including:

- children under 2 years old

- people who have trouble breathing

- People who are unable to remove their own masks" $(4,8)$ ".

\subsection{At-Home management}

If your symptoms are mild enough that you can recover at home, you should:

- Rest- It can make you feel better and may speed your recovery.

- Stay home- Don't go to work, school, or public places.

- Drink fluids-You lose more water when you're sick. Dehydration can make symptoms worse and cause other health problems. 
- Monitor. If your symptoms get worse, call your doctor right away. Don't go to their office without calling first. They might tell you to stay home, or they may need to take extra steps to protect staff and other patients.

- Ask your doctor about over-the-counter medicines that may help, like acetaminophen to lower your fever.

The most important thing to do is to avoid infecting other people, especially those who are over 65 or who have other health problems.

That means:

- Try to stay in one place in your home. Use a separate bedroom and bathroom if you can.

- Tell others you're sick so they keep their distance.

- Cover your coughs and sneezes with a tissue or your elbow.

- Don't share dishes, cups, eating utensils, towels, or bedding with anyone else.

- Clean and disinfect common surfaces like doorknobs, counters, and tabletops“(1)".

\subsection{Preliminary evaluation of Hospitals}

If your case is severe, members of the medical staff will check for signs that the illness is causing more serious problems. They might:

- Check the levels of oxygen in your blood with a clip-on finger monitor

- Listen to your lungs

- Give you a COVID-19 test. This involves putting a 6-inch cotton swab up both sides of your nose for about 15 seconds.

- Give you a chest X-ray or CT scan.

You may get extra oxygen through two small tubes that go just inside your nostrils. In very serious cases, doctors will connect you to a machine that can breathe for you, called a ventilator.

You may also get fluids through a tube, or I.V, in your arm to keep you from getting dehydrated. Doctors will also closely monitor your breathing. The goal is for your infection to run its course and for your lungs to heal enough that they can breathe on their own again “( 9$)^{\prime \prime}$.

\section{Treatment of COVID-19}

There's currently no treatment specifically approved for COVID-19, and no cure for an infection, although treatments and vaccines are currently under study. Instead, treatment focuses on managing symptoms as the virus runs its course.

- Multiple anti-viral regimens are being tried to help patients with severe symptoms of the virus.

- The FDA has rescinded its emergency authorization for the use of hydroxychloroquine and chloroquine to treat people who are hospitalized with COVID-19.

- Immunomodulatory Agents

- Blood plasma transfusions

- Cardiovascular medicines

- KabaSuraKudineer.

\subsection{Anti-viral or retro-viral medications}

Remdesivir: formally known as GS-5734, is a monophosphate pro-drug that undergoes metabolism to an active Cadenosine nucleoside triphosphate analogue. It was originally to treat Ebola infections (against which it has not been proven effective), but has also shown efficacy against MERS viruses in the laboratory. In several clinical trials, the medicine has been able to reduce the duration of disease by several days in subjects at certain stages of the disease"(10)".

Favipiravir (= favilavir): Favipiravir, previously known as T-705, is a pro-drug of a purine nucleotide, favipiravir ribofuranosyl-5'-triphosphate. Initially only had approval for flu therapy and is only intended for second-line therapy 
in when other flu medications are not or insufficiently effective. After corresponding studies, a medicine based on it was approved in Covid-19 therapy“(10)".

Oseltamivir: It a pro-drug that is metabolized by plasma and hepatic esterase to the active form of oseltamivir carboxylate. It inhibition of neuraminidase results in viral aggregation at the surface of the cell and decreases virus spread within the respiratory tract"(11)".

Lopinavir/ritonavir: it is a protease inhibitors. It treatment of human immunodeficiency virus (HIV) infection since 2000. The SARS-CoV-2 virus is a single-stranded RNA Beta coronavirus. The 3-chymotrypsin-like protease (3CLpro) enzyme plays an important role in processing the viral RNA“(11)".

\subsection{Chloroquine and Hydroxychloroquine}

Chloroquine, a synthetic 4-aminoquinoline, has been known since 1934 as an effective, antimalarial substitute of quinine. It inhibits the glycosylation of cellular receptors of SARS-CoV, the ACE2. Chloroquine effectively reduces the number of infected cells on primate (Vero E6) cell culture infected with SARS-CoV. Hydroxychloroquine is a chloroquine derivative and many similar characteristics, however it has a better safety profile, especially in long-term use. "(12, 13)".

\subsection{Immunomodulatory Agents}

Immune reactions are generally desirable in infected persons; they must not be so excessive that they cause more damage to the lungs than they help. Such an excessive immune reaction is called "cytokine storm" because large amounts of messenger substances called cytokines are released. Several projects on Covid-19 therapy therefore focus on attenuating such an immune reaction with a suitable immunomodulator" $(10)^{\prime \prime}$.

\subsection{Corticosteroids}

Steroid therapy may reduce the mortality rate of severe pneumonia“ $(14,15)$ ", for examples corticosteroids mainly administered as prednisolone 40-50 mg/day or hydrocortisone $240 \mathrm{mg} /$ day. However, COVID-19 pneumonia with organ failure almost always meets the diagnostic criteria for acute respiratory distress syndrome (ARDS). The conventional corticosteroid dose for ARDS is methylprednisolone $1-2 \mathrm{mg} / \mathrm{kg} / \mathrm{day}$ “(16,17)". In addition, dexamethasone $20 \mathrm{mg}$ (corresponding to about $80 \mathrm{mg}$ methylprednisolone) has recently been reported to reduce mortality in ARDS. Moreover, high-dose methylprednisolone (1-2 mg/kg) may be an option"(18)".

Interferons: A group of other immune modulators has already been tested in various studies. They are genetically engineered variants of the body's own messenger substances. It was several subgroups, of which the alpha and beta interferons are important against SARS-CoV-2“(7)”.

\subsection{Blood plasma administration}

Sometimes, doctors administer blood plasma of a person already recovered from SARS. This treatment provides a positive boost to the immune system of the patient"(10)".

\subsection{Cardiovascular medicines}

Against the risk of thrombosis with Covid-19, anticoagulant low-molecular-weight heparins are used.

After retrospective comparisons indicated a possible benefit of acetylsalicylic acid (ASA).Clot-dissolving medicines are also being tested, including the active ingredients Alteplase and Tenecteplase.

Antihypertensive medicines such as telmisartan, valsartan, losartan and candesartan. Dapagliflozine can prevent the occurrence of severe complications such as organ failure in Covid-19 patients.

GSK 'sambrisentan (an endothelin receptor antagonist) is approved for the treatment of pulmonary arterial hypertension.

Currently developing Garadacimab (a factor XIIa inhibitor) as a medicine for hereditary angioedema“(10)". 


\subsection{KabaSuraKudineer}

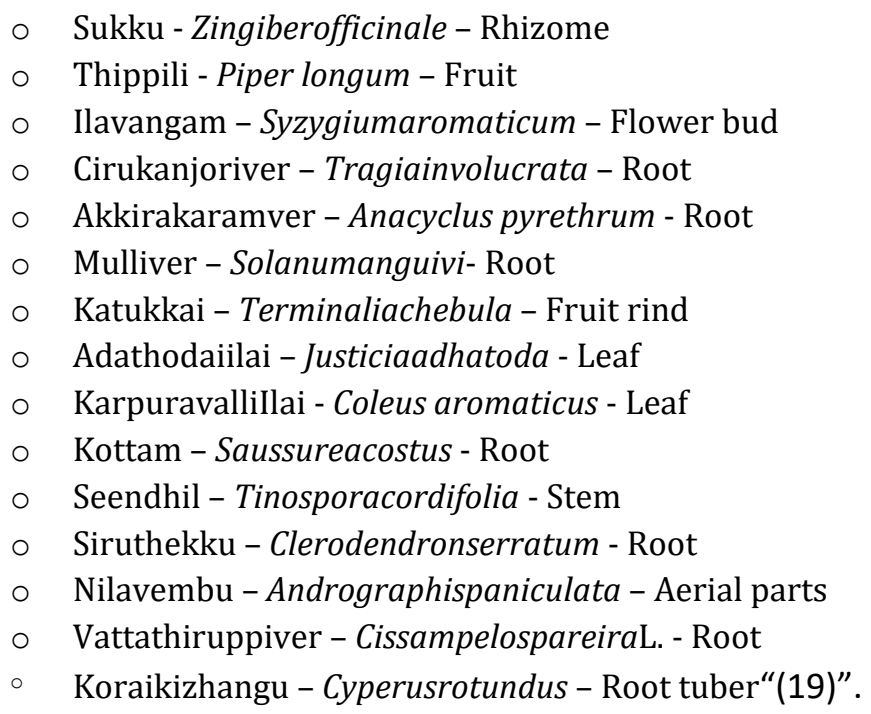

\section{Discussion}

The WHO confirmed that the rational, correct, and consistent use of personal protective equipment (PPE) also helps reduce the spread of pathogens. PPE effectiveness depends strongly on adequate and regular supplies, adequate staff training, appropriate hand hygiene, and appropriate human behavior" $(20)$ ”.

At the level of additional precautions for patients should be practice good hygiene and physical distancing to prevent bacteria and viruses from being transmitted.

The large percentages of the public, these masks can help to slow the transmission of SARS-CoV-2. If your symptoms are mild enough that you can recover at home.

Medicines for lung patients that have been developed, for example, to treat pulmonary fibrosis. They are designed to prevent the patient's lungs from no longer being able to supply the blood with enough oxygen or from not being able to repair themselves properly“(10)”.

Chloroquine and hydroxychloroquine appear to block viral entry into cells by inhibiting glycosylation of host receptors, proteolytic processing, and endosomal acidification. These agents also have immunomodulatory effects through attenuation of cytokine production and inhibition of autophagy and lysosomal activity in host cells"(21)".

Antiviral medicines originally developed for Ebola, hepatitis C, influenza, SARS or MERS (two other coronavirus diseases). They are designed to prevent the viruses from entering or multiplying in body cells.

Cardiovascular medicines, which have been developed to treat blood clots or heart disease, for example. They are designed to prevent complications from Covid-19 disease.

Immunomodulators, which have been developed e.g. against rheumatoid arthritis or inflammatory bowel diseases. They are designed to limit the body's defensive reactions in severe lung infestation so that they do not cause more damage than the viruses themselves

Blood plasma administration: This treatment provides a positive boost to the immune system of the patient "(10)".

Kaba sura kudineer: An AYUSH ministry circular stating guidelines for Siddha practitioners for COVID-19 prevention also recommends consumption of Kaba sura Kudineer twice a day. Phyto-compounds ingredients used in Kaba sura Kudineer act like a molecule binding itself to the spike protein of the virus. This prevents the virus from binding with the receptor proteins in human cells which act as a doorway for the virus to enter our body"(21)". 


\section{Conclusion}

COVID-19 is a new disease caused by the new strain of coronavirus, SARS-CoV-2. Currently, there is no evidence to recommend any specific anti- COVID-19 treatment.

The management of COVID-19 at this moment is based on small-scale clinical studies, which are not sufficient to draw strong conclusions about their efficacy and safety. Based on this clinical pharmacology review, the decision to use these drugs during the COVID-19 pandemic must take into consideration the potential benefits and risks to the patient; the likelihood of the drug to be effective, available, affordable, with the lowest risk for the patients and the community.

\section{Compliance with ethical standards}

\section{Acknowledgments}

The author likes to thank my mentor and Guide Dr. Ajithiadas Aruna for supporting this work.

\section{Disclosure of conflict of interest}

All the authors hereby disclose no conflict of interest.

\section{References}

[1] World Health Organization. Coronavirus disease (COVID- 19).

[2] Monajjemi M, Shahriari S, Mollaami F. Evaluation of Coronavirus Families \& Covid-19 Proteins: Molecular Modeling Study. Bio interface Res. Appl. Chem. 2020; 10: 6039-6057.

[3] Chatterjee S, Chatterjee S. COVID-19 Diagnosis, Treatments and Prevention: A Review. Journal of Advances in Medicine. 2020; 17-34.

[4] Angham GH, Mohammed K, NanyHairunisa, EmadYousif, Salam AM. A Review on COVID -19: Origin, Spread, Symptoms, Treatment and Prevention. Biointerface Research in Applied Chemistry. 2020; 7234-7242.

[5] Lin Ang, Eunhye Song, Hye Won Lee,MyeongSoo Lee. Herbal Medicine for the Treatment of Coronavirus Disease 2019 (COVID-19): A Systematic Review and Meta-Analysis of Randomized Controlled Trials. Journal of Clinical Medicine. 2020; 1-20.

[6] Ramphul K, Mejias S. Coronavirus Disease: A Review of a New Threat to Public Health. Cureus. $12(3): 7276$.

[7] Coronavirus disease 2019 (COVID-19). Accessed: March 11, 2020: https://www.uptodate.com/contents/coronavirus-disease-2019-covid-19\#H2325386707.

[8] Brunilda Nazario MD. WebMD Medical Reference Reviewed. October 23, 2020.

[9] World Health Organization, Minimum requirements for infection prevention and control programmes, World Health Organization. 2019.

[10] www.vfa.de/de/english - inhalte. Therapeutic medicines against the coronavirus infection Covid-19. 11. November 2020.

[11] Jefman E fendi, Marzuki H, Yolanda Safitri, Ferina Angelia. Antiviral treatment of COVID-19: a clinical pharmacology narrative review Article in Medical Journal of Indonesia.2020.

[12] Vinetz JM. Chemotherapy of malaria. In: Brunton LL, Hilal- Dandan R, Knollmann BJ, editors. Goodman \& Gilman's: the pharmacological basis of therapeutics 13th ed. New York: McGraw-Hill Education. 2018; 976-83.

[13] Vincent MJ, Bergeron E, Benjannet S, Erickson BR, Rollin PE, Ksiazek TG, et al. Chloroquine is a potent inhibitor of SARS coronavirus infection and spread. Virol J. 2005; 2: 69.

[14] Stern A, Skalsky K, Avni T, Carrara E, Leibovici L, Paul M. Corticosteroids for pneumonia. Cochrane Database Syst Rev. 2017; 12:CD007720.

[15] Confalonieri M, Urbino R, Potena A, Piattella M, Parigi P, Puccio G, Della Porta R, Giorgio C, Blasi F, Umberger R, Meduri GU. Hydrocortisone infusion for severe community-acquired pneumonia: a preliminary randomized study. Am J RespirCrit Care Med. 2005; 171:242-248. 
[16] Hashimoto S, Sanui M, Egi M, Ohshimo S, Shiotsuka J, Seo R, Tanaka R, Tanaka Y, Norisue Y, Hayashi Y, Nango E, ARDS clinical practice guideline committee from the Japanese Society of Respiratory Care Medicine and the Japanese Society of Intensive Care Medicine. The clinical practice guideline for the management of ARDS in Japan. J Intensive Care. 2017; 5:50.

[17] Meduri GU, Golden E, Freire AX, Taylor E, Zaman M, Carson SJ, Gibson M, Umberger R. Methylprednisolone infusion in early severe ARDS: results of a randomized controlled trial. Chest. 2007; 131:954-963.

[18] Villar J, Ferrando C, Martínez D, et al. Dexamethasone treatment for the acute respiratory distress syndrome: a multicentre, randomised controlled trial. Lancet Respir Med. 2020; 8:267-276.

[19] Divya RA. SARS-COV-2 and KabasuraKudineer. A Mini review on the antiviral properties of ancient siddha medicine from India. J Thoracic Disease and Cardiothoracic Surgery. 2020; 1(2).

[20] World Health Organization. Coronavirus disease (COVID- 19) technical guidance: Infection prevention and control / WASH. March 2020.

[21] James M, Sanders, Marguerite L, Monogue, Tomasz Z, Jodlowski, James B, Cutrell. Pharmacologic Treatments for Coronavirus Disease 2019 (COVID-19) A Review. Clinical Review \& Education. 2020; 1824 -1839. 